\title{
INTEGRATION OF REAL-VALUED SET FUNCTIONS IN ABSTRACT SPACES
}

\author{
P. D. FINCH \\ (received 16 August 1963)
}

\section{Introduction}

Let $\mathscr{X}$ an abstract space of points $x$ and let $\mathscr{M}$ be a $\sigma$-field of subsets of $\mathscr{X}$, that is a class of subsets of $\mathscr{X}$ such that (i) $\mathscr{X} \in \mathscr{M}$, (ii) if $M \in \mathscr{M}$ then $\mathscr{X}-M \in \mathscr{M}$ and (iii) if $\left\{M_{j}\right\}$ is any sequence of elements of $\mathscr{M}$ then $\bigcup_{i} M_{j} \in \mathscr{K}$.

We suppose that a $\sigma$-finite measure $\mu(\cdot)$ is defined on $\mathscr{M}$, that is, $\mu(M)$ is a real-valued function defined for each $M \in \mathscr{M}$ such that (i) $\mu(M) \geqq 0$ (ii) $\mu\left(\sum_{j} M_{j}\right)=\sum_{j} \mu\left(M_{j}\right)$ for each sequence of mutually disjoint elements of $\mathscr{K}$, and (iii) there is at least one sequence $\left\{M_{i}\right\}$ of mutually disjoint elements of $\mathscr{M}$ such that $\sum_{j} M_{j}=\mathscr{X}$ and $\mu\left(M_{j}\right)<\infty$ for each $j$.

By a partition $\Pi(S)$ of an element $S \in \mathscr{M}$ we mean a finite or countable system of mutually disjoint elements of $\mathscr{H}$ whose union is $S$ and such that $\mu(M)<\infty$ for each $M \in \Pi(S)$.

If $\Pi_{1}(S)=\left\{M_{1 i}\right\}, \Pi_{2}(S)=\left\{M_{2 j}\right\}, i, j \geqq 0$ are two partitions of $S$ then their intersection, denoted by $\Pi_{1} \cdot \Pi_{2}(S)$ is the partition of $S$ consisting of the elements of the sequence $\left\{M_{1 i} M_{2 j}\right\}, i, j \geqq 0$. This definition extends readily to the intersection $\Pi_{j} \Pi_{3}(S)$ of a finite system of partitions of $S$. If $\Pi(S), \Pi_{1}(S)$ are two partitions of $S$ such that each element of $\Pi(S)$ which has positive $\mu$-measure is a subset of an element of $\Pi_{1}(S)$ then we say that $\Pi(S)$ is finer than $\Pi_{1}(S)$ and write $\Pi(S) \geqq \Pi_{1}(S)$. Evidently $\bigcap_{j=1}^{k} \Pi_{j}(S) \geqq \Pi_{j^{\prime}}(S)$ for each $j^{\prime} \geqq 1$, and $k>1$.

For any $S \in \mathscr{K}$ we denote by $\mathscr{K}(S)$ the $\sigma$-field of all elements $M \in \mathscr{M}$ such that $M \subset S$.

Let $F(\Pi)$ be a real-valued function on the partitions $\Pi=\Pi(S)$ of a fixed element $S \in \mathscr{M}$. We write

$$
F=(I) \lim F(\Pi)
$$

if, to every $e>0$ there is a partition $\Pi$, of $S$ such that

$$
|F-F(I)|<e
$$

for all partitions $\Pi$ of $S$ such that $\Pi \geqq \Pi_{e}$.

When the $\Pi$-limit exists it is unique, that is, if there exists also an $F^{\prime}$ 
such that to every $e>0$ there is a partition $\Pi_{e}^{\prime}$ of $S$ which is not finer than $\Pi_{e}$ and such that

$$
\left|F^{\prime}-F\left(\Pi^{\prime}\right)\right|<e
$$

for all partitions $\Pi^{\prime}$ of $S$ such that $\Pi^{\prime} \geqq \Pi_{e}^{\prime}$, then since

$$
\left|F-F^{\prime}\right| \leqq\left|F-F\left(\Pi^{\prime \prime}\right)\right|+\left|F^{\prime}-F\left(\Pi^{\prime \prime}\right)\right|<2 e
$$

for all $\Pi^{\prime \prime} \geqq \Pi_{e}^{\prime \prime}=\Pi_{e} \cdot \Pi_{e}^{\prime}$, we have $F^{\prime}=F$.

The $\Pi$-limit introduced above is a limit in the sense of Moore-Smith convergence, see, for example Kelley [6].

Let $f(\cdot)$ be a real-valued function on the elements of $\mathscr{M}$. In sections $2-6$ below we construct a theory of integration of $f(\cdot)$ with respect to the measure $\mu$ by writing

$$
(\Pi) \int_{\mu(s)} f(\cdot) \mu(\cdot)=(\Pi) \lim \Sigma_{\Pi(S)} f(M) \mu(M)
$$

where the summation is over all elements of the partition $\Pi(S)$ of $S$, whenever the $I I$-limit exists. The need for such a theory arises in a number of contexts in the theory of probability, for example Finch [2]. Applications to the theory of probability will be discussed in later papers. I am indebted to J. E. Moyal for suggesting that Moore-Smith convergence could be an appropriate tool in the analysis of the probability problems which gave rise to the theory discussed below. The results of this paper are a direct consequence of that suggestion.

In what follows we restrict attention to integration over the $\sigma$-field of $\mu$-measurable subsets of $\mathscr{X}$. There is no difficulty, however, in extending the results to an arbitrary subset of $\mathscr{X}$. This extension may be carried out by introducing the Carathéodory outer measure

$$
\mu^{*}(X)=\inf \{\mu(M) ; X \subset M, M \in \mathscr{M}\}
$$

for any subset $X \subset \mathscr{X}$.

Following Carathéodory a subset $M^{*} \subset \mathscr{X}$ is said to be $\mu^{*}$-measurable if

$$
\mu^{*}(X)=\mu^{*}\left(X M^{*}\right)+\mu^{*}\left(X\left(\mathscr{X}-M^{*}\right)\right)
$$

for each $X \subset \mathscr{X}$.

If $\mathscr{H}^{*}$ denotes the class of all $\mu^{*}$-measurable sets then $\mathscr{H} \subset \mathscr{H}^{*}$ and $\mathscr{H}^{*}$ is a $\sigma$-field of subsets of $\mathscr{X}$. As is well-known, $\mu^{*}$ is countably additive over the $\sigma$-field $\mathscr{M}^{*}(S)$ of all sets of the form $M^{*} S, M^{*} \in \mathscr{M}^{*}$. If we define a partition of an arbitrary $S \subset \mathscr{X}$ to be a finite or countable system of mutually disjoint elements of $\mathscr{K}^{*}(S)$ whose union is $S$ and such that $\mu^{*}(X)<\infty$ for each element $X$, of the partition then the method and results below remain valid.

The integration theory below may be extended also to signed measures 
in an obvious way by means of the Hahn-decomposition theorem.

The theory of this paper is similar to a modification of the theory of Birkhoff [1] suggested by Hildebrandt [5] and derives from an early work paper of Fréchet [4] which uses ideas due to W. H. Young. For an account of these and other theories of integration we refer to the review paper of Hildebrandt [5].

The theory below is, of course, much less general than that of Birkhoff since we consider only real-valued functions whereas Birkhoff considers functions taking values in a Banach space. However, the emphasis of this paper is rather different from that of other authors whose main interest has been the integration of point functions. In this paper the interest centres on the integration of set functions. However, in section 8 , we show how the present theory may be applied to integrate measurable point functions.

In a later paper we examine the extent to which the methods of this paper can be used when the set functions take values in a metric vector space. Finally we remark that the theory of this paper could have been developed by replacing the $\sigma$-field $\mathscr{H}$ by a Boolean $\sigma$-algebra containing a null and an all element. However, with specific applications to probability theory in mind it seemed preferable to pursue the method adopted below.

\section{Set functions on $\mathscr{K}$}

By a set function $f(\cdot)$ on $\mathscr{H}$ we mean a real-valued function $f(M)$ defined for each $M \in \mathscr{M}$. However, we consider as equivalent two set functions $f_{1}, f_{2}$ such that $f_{1}(M) \neq f_{2}(M)$ implies $\mu(M)=0$. Two equivalent set functions will be said to be equal almost everywhere with respect to $\mu$ or in abbreviation, equal a.e. $\mu$. Instead of talking of an equivalence class of set functions we adopt the convention that a set function can be defined arbitrarily on the elements of $\mathscr{K}$ with zero $\mu$-measure.

If $S \in \mathscr{W}$ has positive $\mu$-measure, a set function $f(\cdot)$ is said to be essentially bounded on $\mathscr{H}(S)$ if there is a partition $\Pi_{f}$ of $S$ such that $f(\cdot)$ is bounded on the family of all elements with positive $\mu$-measure of all partitions of $S$ which are finer than $\Pi_{f}$.

For each element $M \in \mathscr{H}$ with $\mu(M)>0$ we define

$$
\left\{\begin{array}{l}
f^{(+)}(M)=\sup \left\{f\left(M_{1}\right) ; M_{1} \subset M, M_{1} \in \mathscr{M}, \mu\left(M_{1}\right)>0\right\} \\
f^{(-)}(M)=-\inf \left\{f\left(M_{1}\right) ; M_{1} \subset M, M_{1} \in \mathscr{M}, \mu\left(M_{1}\right)>0\right\} .
\end{array}\right.
$$

When $M \in \mathscr{M}$ and $\mu(M)=0$ it is convenient to adopt the convention

$$
f^{(-)}(M)=f(M)=f^{(+)}(M)=0 .
$$


The set functions $f^{(+)}(\cdot), f^{(-)}(\cdot)$ are called the upper and lower variations, respectively, of $f(\cdot)$ on $\mathscr{H}$ with respect to $\mu$. We write

$$
|f|(M)=f^{(+)}(M)+f^{(-)}(M) .
$$

The set function $|f|(\cdot)$ is called the total variation of $f(\cdot)$ on $\mathscr{M}$ with respect to $\mu$. If $M_{1}, M_{2} \in \mathscr{M}, M_{1} \subset M_{2}$ and $\mu\left(M_{1}\right)>0$ then

$$
-f^{(-)}\left(M_{2}\right) \leqq-f^{(-)}\left(M_{1}\right) \leqq f\left(M_{1}\right) \leqq f^{(+)}\left(M_{1}\right) \leqq f^{(+)}\left(M_{2}\right)
$$

and hence

$$
|f|\left(M_{1}\right) \leqq|f|\left(M_{2}\right)
$$

We prove now

LemmA (2.1). If $M \in \mathscr{M}, \mu(M)>0$ and $\left|f^{(+)}(M)\right|<\infty$ then to every $\delta>0$ there is a $M_{\delta} \in \mathscr{M}, \mu\left(M_{\delta}\right)>0$ and $M_{\delta} \subset M$ such that

$$
0 \leqq f^{(+)}\left(M_{\delta}\right)-f\left(M_{\delta}\right)<\delta .
$$

Proof. By the definition of $f^{(+)}(M)$ there is an $M_{\delta} \subset M, \mu\left(M_{\delta}\right)>0$ $M_{\delta} \in \mathscr{A}$ such that

$$
f^{(+)}(M)-\delta \leqq f\left(M_{\delta}\right) \leqq f^{(+)}\left(M_{\delta}\right) \leqq f^{(+)}(M) .
$$

This proves the lemma.

Using Lemma (2.1) and a result of Finch [3] we prove the important

THEOREM (2.1). If $M \in \mathscr{M}, 0<\mu(M)<\infty$ and if $f(\cdot)$ is bounded on $\mathscr{M}(M)$ then to every $\delta>0$ there is a sequence $\left\{M_{\delta}^{n}\right\}, n \geqq 0$ of mutually disjoint subsets of $M$, each of which belongs to $\mathscr{M}$ and such that

(i) $\mu\left(M_{\delta}^{0}\right)=0, \mu\left(M_{\delta}^{n}\right)>0, \quad n>0$,

(ii) $0 \leqq f^{(+)}\left(M_{\delta}^{n}\right)-f\left(M_{\delta}^{n}\right)<\delta, \quad n>0$,

(iii) $\sum_{n=0}^{\infty} M_{\delta}^{n}=M$.

Proof. Denote by $\mathscr{F}_{\delta}$ the family of all $\mu$-measurable subsets of $M$ which have positive $\mu$-measure and which satisfy condition (ii) of the theorem. By lemma (2.1) this family is not empty. For any element $F \in \mathscr{F}_{\delta}$ we define the $R$-class of $F$ to consist of $F$ and all the elements of $\mathscr{F}_{\delta}$ which are disjoint to $F$. By a result of Finch [3] the family $F_{\delta}$ has a maximal $R$-section, that is, a maximal set of mutually disjoint elements of $\mathscr{F}_{\delta}$. Since $\mu(M)<\infty, \mu(F)>0$ for each $F \in \mathscr{F}_{\delta}$ and $\mu$ is countably additive there can be at most a countable number of elements of $\mathscr{F}_{\delta}$ in a maximal $R$-section. Let these elements be $M_{\delta}^{n}, n \geqq 1$. Then the maximality of the sequence $\left\{M_{\delta}^{n}\right\}, n \geqq 1$ implies that $M_{\delta}^{0}=M-\sum_{n=1}^{\infty} M_{\delta}^{n}$ has $\mu$-measure zero, for otherwise lemma (2.1) implies the existence of an $M_{\delta} \in \mathscr{F}_{\S}$ 
$\mu\left(M_{\delta}\right)>0$ and $M_{\delta} \subset M_{\delta}^{0}$ thus contradicting the maximality of the sequence $\left\{M_{\delta}^{n}\right\}, n \geqq 1$. This completes the proof of the theorem.

The following corollary is important.

Corollary. (2.1). If $S \in \mathscr{M}, f(\cdot)$ is essentially bounded on $\mathscr{M}(S)$ then there is a partition $\Pi$, of $S$ such that to every partition $\Pi$ of $S$ which is finer than $\Pi_{f}$ and to every $\delta>0$ there is a partition $\Pi_{\delta}$ of $S$ such that $\Pi_{\delta} \geqq \Pi$ and

$$
0 \leqq f^{(+)}\left(M_{\delta}\right)-f\left(M_{\delta}\right)<\delta
$$

for each element $M_{\delta} \in \Pi_{\delta}$ which has positive $\mu$-measure.

The corollary is proved easily by taking $\Pi_{f}$ to be the partition occurring in the definition of essential boundedness and by applying theorem (2.1) to each element of the partition $\Pi \geqq \Pi_{f}$ which has positive $\mu$-measure.

In exactly the same way we can prove

CoRollary (2.2). If $S \in \mathscr{M}, f(\cdot)$ is essentially bounded on $\mathscr{H}(S)$ then there is a partition $\Pi_{f}$ of $S$ such that to every partition $\Pi$ of $S$ which is finer than $\Pi_{f}$ and to every $\delta>0$ there is a partition $\Pi_{\delta}$ of $S$ such that $\Pi_{\delta} \geqq \Pi$ and

$$
0 \leqq f\left(M_{\delta}\right)+f^{(-)}\left(M_{\delta}\right)<\delta
$$

for every element $M_{\delta} \in I_{\delta}$ which has positive $\mu$-measure.

\section{Integration of essentially bounded non-negative set functions}

Throughout this section $S$ is an element of $\mathscr{M}$ such that $0<\mu(S)<\infty$, $f(\cdot)$ is a set function which is non-negative and essentially bounded on $\mathscr{M}(S)$. All the partitions $\Pi(S)$ of $S$ which occur in this section will be assumed to be finer than the partition $\Pi_{f}$ which occurs in the definition of essential boundedness. We write

$$
(I I) \int_{M(S)} f(\cdot) \mu(\cdot)=(\Pi) \lim \left[\sum_{n(S)} f(M) \mu(M)\right]
$$

whenever the $\Pi$-limit on the right exists. When the $\Pi$-limit (3.1) exists we say that $f(\cdot)$ is $\Pi$-integrable on $\mathscr{H}(S)$ with respect to $\mu$. The summation occurring in (3.1) is over all elements of the partition $\Pi(S)$ of $S$ and in (3.1) and in similar summations below we adopt the convention (2.2).

In order to obtain conditions under which the $\Pi$-integral exists it is convenient to introduce a Riemann type integral which we define in terms of upper and lower Darboux Sums. Thus we write

$$
\left\{\begin{array}{l}
F_{S}^{(+)}(\Pi)=\sum_{\Pi(S)} f^{(+)}(M) \mu(M) \\
F_{S}(\Pi)=\sum_{\Pi(S)} f(M) \mu(M) \\
F_{S}^{(-)}(\Pi)=\sum_{I I(S)} f^{(-)}(M) \mu(M)
\end{array}\right.
$$


Since $f(\cdot)$ is non-negative and essentially bounded on $\mathscr{H}(S), \mu$ is completely additive and $\mu(S)<\infty$ the series in (3.2) all converge.

From (2.4) we deduce that if $\Pi_{1}(S) \geqq \Pi_{2}(S)$

$$
-F_{S}^{(-)}\left(\Pi_{2}\right) \leqq-F_{S}^{(-)}\left(\Pi_{1}\right) \leqq F_{S}\left(\Pi_{1}\right) \leqq F_{S}^{(+)}\left(\Pi_{1}\right) \leqq F_{S}^{(+)}\left(\Pi_{2}\right)
$$

Since $-F_{S}^{(-)}(\Pi) \leqq \mu(S)$ sup $\{f(\cdot)\}$ and $F_{S}^{(+)}(I) \geqq \mu(S)$ inf $\{f(\cdot)\}$

$$
\left\{\begin{array}{l}
F_{S}=\inf \left\{F_{S}^{(+)}(\Pi)\right\} \\
\underline{F}_{S}=\sup \left\{-F_{S}^{(-)}(\Pi)\right\}
\end{array}\right.
$$

are finite. It follows at once from the monoticity implied by (3.3) that

$$
\underline{F}_{S} \leqq \bar{F}_{S}
$$

and

$$
\left\{\begin{array}{l}
F_{S}=(\Pi) \int_{\mu(S)} f^{(+)}(\cdot) \mu(\cdot) \\
F_{S}=-(\Pi) \int_{\mu(S)} f^{(-)}(\cdot) \mu(\cdot) .
\end{array}\right.
$$

If $F_{S}=F_{S}=F_{S}$ we say that $f(\cdot)$ is $R$-integrable on $\mathscr{H}(S)$ with respect to $\mu$ and write

$$
F_{S}=(R) \int_{\boldsymbol{\mu}(S)} f(\cdot) \mu(\cdot)
$$

The following theorems establish the equivalence of the $R$ and $\Pi$ integrals and lead to a necessary and sufficient condition that the integral exists.

THEOREM (3.1). If $f(\cdot)$ is $R$-integrable on $\mathscr{M}(S)$ with respect to $\mu$ then it is also $\Pi$-integrable on $\mathscr{M}(S)$ with respect to $\mu$ and

$$
(\Pi) \int_{\mu(s)} f(\cdot) \mu(\cdot)=(R) \int_{\mu(s)} f(\cdot) \mu(\cdot) \text {. }
$$

$A$ necessary and sufficient condition that $f(\cdot)$ be R-integrable on $\mathscr{A}(S)$ with respect to $\mu$ is that the $\Pi$-integral of the total variation of $f(\cdot)$ on $\mathscr{M}(S)$ with respect to $\mu$ is zero, that is,

$$
\text { (II) } \int_{\mu(s)}|f|(\cdot) \mu(\cdot)=0 .
$$

Proof. If $f(\cdot)$ is $R$-integrable then $\underline{F}_{S}=F_{S}=F_{S}$. From (3.3) it follows that $(\Pi) \lim F_{S}(I)$ exists and is equal to $F_{S}$. This proves (3.8). When $f(\cdot)$ is $R$-integrable it follows from (3.6) and the additivity of the $\Pi$-limit that (3.9) is true. Conversely when (3.9) is true it follows from (3.6) that $F_{S}=\bar{F}_{S}$ and so $f(\cdot)$ is $R$-integrable.

The next theorem in conjunction with theorem (3.1) establishes the equality of the $I I$ and $R$-integrals. 
THEOREM (3.2). If $f(\cdot)$ is $\Pi$-integrable on $\mathscr{A l}(S)$ with respect to $\mu$ then it is also $R$-integrable and the two integrals have the same value

Proof. If $f(\cdot)$ is $\Pi$-integrable on $\mathscr{M}(S)$ then it follows from (3.6) and the additivity of the $\Pi$-limit that $\left(f^{(+)}(\cdot)-f(\cdot)\right)$ is $\Pi$-integrable. Let $F_{S}$ be the value of the $\Pi$-integral of $f(\cdot)$. Then given $e>0$ there is a partition $\Pi_{e}(S)$ such that

$$
\left|\left(F_{S}-F_{S}\right)-\left(F_{S}^{(+)}(\Pi)-F_{S}(\Pi)\right)\right|<e
$$

for all $\Pi(S) \geqq \Pi_{e}(S)$. But it follows from Corollary (2.1) that given any $\delta>0$ there is a partition $\Pi_{\delta}(S) \geqq \Pi_{\odot}(S)$ such that

$$
0 \leqq F_{S}^{(+)}(\Pi)-F_{S}(\Pi)<\delta \mu(S) \text {. }
$$

It follows at once that $F_{S}=F_{S}$. A similar argument shows that $\underline{F}_{S}=F_{S}$. This proves the theorem.

Finally we prove

THEOREM (3.3). A necessary and sufficient condition that $f(\cdot)$ be $I$ integrable on $\mathscr{N}(S)$ with respect to $\mu$ is that to every $\delta>0$ there exists a partition $\Pi_{\delta}(S)$ such that $|f|(M)<\delta$ for every element $M \in \Pi_{\delta}(S)$ with positive $\mu$ measure.

In order to prove the theorem we require the following

LEMMA (3.1). If $f(\cdot)$ is $\Pi$-integrable on $\mathscr{M}(S)$ with respect to $\mu$ and if $M_{0} \in \mathscr{H}(S)$ has positive and finite $\mu$-measure then

$$
\inf \left\{|f|(M) ; M \subset M_{0}, M \in \mathscr{M}(S), \mu(M)>0\right\}=0 .
$$

Proof. Suppose to the contrary that the infimum in (3.10) takes the value $\delta>0$. Since $f(\cdot)$ is $\Pi$-integrable it is also $R$-integrable and (3.9) is true. Thus there is a partition $\Pi_{\delta}(S)$ such that

$$
\sum_{n(S)}|f|(M) \mu(M)<\delta \mu\left(M_{0}\right)
$$

for all $\Pi(S) \geqq \Pi_{\delta}(S)$.

Let $\Pi_{\delta}(S)=\left\{M_{\delta}^{n}\right\}, n \geqq 0, M_{\delta}^{n} \in \mathscr{K}(S)$ and let $\Pi_{1}(S)$ be the partition $\left\{M_{0}, S-M_{0}\right\}$. Writing $\Pi_{1 \delta}(S)=\Pi_{1} \cdot \Pi_{\delta}(S)$ we have

$$
\begin{aligned}
\sum_{\pi_{1 d}(S)}|f|(M) \mu(M) & \geqq \sum_{n=0}^{\infty}|f|\left(M_{\delta}^{n} M_{0}\right) \mu\left(M_{\delta}^{n} M_{0}\right) \\
& \geqq \delta \mu\left(M_{0}\right) .
\end{aligned}
$$

But $\Pi_{1 \delta}(S) \geqq \Pi_{\delta}(S)$ and the inequality above contradicts (3.11). Thus (3.10) is true and the lemma is proved.

Proof of Theorem (3.3). (a) Sufficiency. Under the hypothesis 
of the theorem it follows from (2.5) that $|f|(M)<\delta$ for all elements $M \in \Pi(S) \geqq \Pi_{\delta} S$ with positive $\mu$-measure. Thus

$$
\sum_{n(S)}|f|(M) \mu(M) \leqq \delta \mu(S)
$$

for all $\Pi(S) \geqq \Pi_{\delta}(S)$. Thus (3.9) is true and $f(\cdot)$ is $R$-integrable, hence also $\Pi$-integrable.

(b) Necessity. If $f(\cdot)$ is $\Pi$-integrable on $\mathscr{H}(S)$ with respect to $\mu$ then it follows from lemma (3.1) that to each $\delta>0$ and each element $M \in \mathscr{H}(S)$ with $\mu(M)>0$ there is an element $M_{\delta} \in \mathscr{M}, \mu\left(M_{\delta}\right)>0$, and $M_{\delta} \subset M$ such that $|f|\left(M_{\delta}\right)<\delta$. An argument similar to that used to prove corollary (2.1) establishes that to every $\delta>0$ and every partition $\Pi(S)$ of $S$ there is a partition $\Pi_{\mathrm{d}}(S) \geqq \Pi(S)$ such that $|f|(M)<\delta$ for each $M \in \Pi_{\delta}(S)$ with positive $\mu$-measure. This completes the proof of the theorem.

A set function which satisfies the conditions of theorem (3.3) will be said to be regular on $\mathscr{H}(S)$ with respect to $\mu$.

\section{Integration unbounded non-negative set functions}

In this section $S$ is again an element of $\mathscr{H}$ such that $0<\mu(S)<\infty$, $f(\cdot)$ is a set function which is non-negative but unbounded on $\mathscr{H}(S)$. We write

and define

$$
\text { (n) } f(M)=\left\{\begin{array}{cll}
f(M) & \text { if } & f(M) \leqq n \\
n & \text { if } & f(M)>n
\end{array}\right.
$$

$$
\text { (I) } \int_{\mu(s)} f(\cdot) \mu(\cdot)=\lim _{n \rightarrow \infty} \int_{\mu(s)}{ }^{(n)} f(\cdot) \mu(\cdot)
$$

whenever ${ }^{(n)} f(\cdot)$ is regular on $\mathscr{k}(S)$ with respect to $\mu$ for each $n>0$ and the limit on the right of (4.2) exists.

When $f(\cdot)$ is unbounded but essentially bounded on $\mathscr{H}(S)$ it is clear that (4.2) yields the same integral as the last section.

\section{Integration of unrestricted set functions}

Let $S \in \mathscr{K}, 0<\mu(S)<\infty$ and let $f(\cdot)$ be a set function on $\mathscr{H}(S)$ which is not restricted to non-negative values and is not necessarily essentially bounded. We write

$$
\begin{aligned}
& f_{+}(M)=\left\{\begin{array}{ccc}
f(M) & \text { if } & f(M) \geqq 0 \\
0 & \text { if } & f(M)<0
\end{array}\right. \\
& f_{-}(M)=\left\{\begin{array}{ccc}
0 & \text { if } & f(M) \geqq 0 \\
-f(M) & \text { if } & f(M)>0
\end{array}\right.
\end{aligned}
$$


so that

$$
f(M)=f_{+}(M)-f_{-}(M)
$$

We define the $\Pi$-integral of $f(\cdot)$ on $\mathscr{M}(S)$ with respect to $\mu$ by

$$
(I) \int_{\mu(s)} f(\cdot) \mu(\cdot)=(I) \int_{\mu(s)} f_{+}(\cdot) \mu(\cdot)-(I) \int_{\mu(s)} f_{-}(\cdot) \mu(\cdot)
$$

whenever the $\Pi$-integrals on the right exist, in which case we say that $f(\cdot)$ is $\Pi$-integrable on $\mathscr{H}(S)$ with respect to $\mu$.

It follows at once that if $f(\cdot)$ is $\Pi$-integrable then $|f(\cdot)|$ is also $\Pi$-integrable and

$$
\left|(\Pi) \int_{\mu(s)} f(\cdot) \mu(\cdot)\right| \leqq(\Pi) \int_{\mu(s)}|f(\cdot)| \mu(\cdot) .
$$

Finally if $\mu(S)=0$ we define

$$
(I) \int_{\mu(S)} f(\cdot) \mu(\cdot)=0 .
$$

\section{Integration on sets with infinite $\mu$-measure}

Let $\left\{S_{j}\right\}, j \geqq 1$ be a sequence of mutually disjoint elements of $\mathscr{A}$ such that for each $j \geqq 1,0<\mu\left(S_{f}\right)<\infty$ and write $S=\sum_{j=1}^{\infty} S_{f}$. We do not assume that $\sum_{j=1}^{\infty} \mu\left(S_{j}\right)<\infty$ and so $S$ may have infinite $\mu$-measure. For each $j \geqq 1$ let $\Pi\left(S_{j}\right)=\left\{M_{j}^{n}\right\}, n \geqq 0$ be a partition of $S_{f}$, we define the sum of the partitions $\Pi\left(S_{f}\right)$,

$$
\Pi(S)=\sum_{j=1}^{\infty} \Pi\left(S_{j}\right)
$$

to be the partition of $S$ consisting of the double sequence $\left\{M_{j}^{n}\right\}$ of elements of $\mathscr{M}(S)$.

Let $S$ be an arbitrary element of $\mathscr{H}$. Since $\mu$ is $\sigma$-finite on $\mathscr{M}$ there exists a sequence of mutually disjoint elements of $\mathscr{M},\left\{S_{j}\right\}, j \geqq 0$ such that $\mu\left(S_{0}\right)=0,0<\mu\left(S_{j}\right)<\infty, j>0$ and $S=\sum_{j=0}^{\infty} S_{j}$. Let $f(\cdot)$ be a $\Pi$-integrable set function on $\mathscr{M}\left(S_{j}\right)$ for each $j \geqq 1$, then we define

$$
(\Pi) \int_{\mathcal{M}(S)} f(\cdot) \mu(\cdot)=\sum_{j=1}^{\infty}\left(\Pi^{\prime}\right) \int_{\mathcal{M}\left(S_{j}\right)} f(\cdot) \mu(\cdot)
$$

whenever the series on the right converges.

We show now that this definition does not depend on the particular partition $\left\{S_{i}\right\}, j \geqq 0$ of $S$ which occurs in (6.1). Explicitly we prove

THEOREM (6.1). Let $S \in \mathscr{H}$. If $f(\cdot)$ is a set function on $\mathscr{H}$ which is $\Pi$ integrable on each element of $\mathscr{M}(S)$ with finite $\mu$-measure then 


$$
(I I) \int_{\mu(S)} f(\cdot) \mu(\cdot)=(I I) \lim \left[\sum_{\Pi(S)} f(M) \mu(M)\right]
$$

in the sense that if either side exists then so does the other and the two are equal.

PRoof. Since $\mu$ is $\sigma$-finite there is a partition $\left\{S_{i}\right\}, j \geqq 0$ of $S$ such that $\mu\left(S_{0}\right)=0,0<\mu\left(S_{f}\right)<\infty, j>0$. Write

$$
F_{s}(I T)=\sum_{\Pi\left(S_{j}\right)} f(\cdot) \mu(\cdot), \quad j>0
$$

for any partition $\Pi\left(S_{j}\right)$ of $S_{j}$. By the hypothesis of the theorem $F_{j}=(I) \lim F_{j}(I I)$ exists. Thus to each $j \geqq 1$ and each $e>0$ there is a partition $\Pi_{e}\left(S_{f}\right)$ such that

$$
\left|F_{j}-F_{j}(\Pi)\right|<e / 2^{j}
$$

for all $\Pi=\Pi\left(S_{j}\right) \geqq \Pi_{0}\left(S_{j}\right)$. Let

$$
\Pi_{\bullet}(S)=\sum_{j=1}^{\infty} \Pi_{\bullet}\left(S_{j}\right)
$$

be the sum of the partitions $\Pi_{e}\left(S_{f}\right)$. Then

$$
\sum_{j=1}^{\infty}\left|F_{j}-F_{j}(\Pi)\right|<e
$$

for all partitions $\Pi=\Pi(S) \geqq \Pi_{e}(S)$.

The statement of the theorem follows from (6.3) by a standard argument. Note that the uniqueness of the $\Pi$-limit, when it exists, established in section 1, ensures that the definition (6.1) does not depend on the particular defining sequence $\left\{S_{\}}\right\}$.

Note also that $(6.1)$ is valid when $\sum \mu\left(S_{j}\right)<\infty$. We can express the results of this section therefore by the remark that the $\Pi$-integral is a completely additive set function on $\mathscr{K}$.

\section{Integration of point functions}

By a choice function on $\mathscr{K}$ we mean a relation which associates to each element $M \in \mathscr{M}$ a point $\xi(M) \in \mathscr{X}$. If $\xi(M) \in M$ for each $M \in \mathscr{M}$ we say that $\xi(\cdot)$ is a restricted choice function. The existence of a choice function is ensured by the axiom of choice.

If $f(x)$ is a real-valued point function on $\mathscr{X}$ and if $\xi(\cdot)$ is a choice function on $\mathscr{M}$ then we can associate a set function on $\mathscr{K}$ to the point function by means of the equation

$$
f_{\xi}(M)=f(\xi(M))
$$


If

$$
\left\{\begin{array}{l}
S(M)=\sup \{f(x): x \in M\} \\
i(M)=\inf \{f(x): x \in M\}
\end{array}\right.
$$

then for any restricted choice function $\xi(\cdot)$ we have

$$
i(M) \leqq f_{\xi}(M) \leqq S(M) .
$$

The set function $S(M)$ is monotonic non-decreasing, that is, $S\left(M_{1}\right) \leqq S\left(M_{2}\right)$ when $M_{1} \subset M_{2}$. It follows that for $\mu(M)>0, S^{(+)}(M)=S(M)$ and hence that $|S|(M)=S(M)+S^{(-)}(M)$. Thus, by corollary (2.2) and theorem (3.3), $S(M)$ is $\Pi$-integrable on any set $S$ of finite $\mu$-measure such that $S(\cdot)$ is essentially bounded on $\mathscr{H}(S)$. Similarly $i(M)$ is $\Pi$-integrable.

Suppose, for simplicity, that $\mu(\mathscr{X})<\infty, 0 \leqq f(x)<1, x \in \mathscr{X}$. If $f(x)$ is measurable with respect to $\mathscr{L}$ that is if the sets $F_{a}=\{x: f(x)>a\}$ belong to $\mathscr{A}$ for each real $a$ it follows that the sets

$$
M_{j, n}=\{x:(j-1) / n \leqq f(x)<j / n\}, \quad 1 \leqq j \leqq n,
$$

are elements of $\mathscr{H}$ and that for each $n \geqq 1,\left\{M_{j, n}\right\}, 1 \leqq j \leqq n$ is a partition of $\mathscr{X}$. From (7.3) the total variation of $f_{E}(\cdot)$ does not exceed $1 / n$ on any element of this partition whatever the restricted choice function $\xi(\cdot)$. It follows from theorem (3.3) that $f_{E}(\cdot)$ is $\Pi$-integrable on $\mathscr{M}$. From (7.3) we obtain

$$
(\Pi) \int_{\mathcal{M}} i(\cdot) \mu(\cdot) \leqq(\Pi) \int_{\mathcal{M}} f_{\xi}(\cdot) \mu(\cdot) \leqq(\Pi) \int_{\mathcal{M}} S(\cdot) \mu(\cdot) .
$$

Since $f(x)$ is measurable $\not t$ the extreme expressions in the above inequality are just the lower and upper Lebesque integrals of $f(x)$ with respect to the measure $\mu$, they are therefore equal. Thus the $\Pi$-integral on $\boldsymbol{l}$ of the set function associated to the bounded measurable point function $f(x)$, by an arbitrary restricted choice function is just the Lebesque integral of $f(x)$ with respect to the measure $\mu$.

Conversely if the extreme expressions in the inequalities (7.4) are equal $f_{\xi}(\cdot)$ is $\Pi$-integrable for any restricted choice function $\xi(\cdot)$ and the value of the integral does not depend on $\xi(\cdot)$. Further to any $\delta>0$ there is a partition $\Pi_{\delta}$ of $\mathscr{X}$ such that

$$
\left|f\left(x_{1}\right)-f\left(x_{2}\right)\right|<\delta
$$

for all $x_{1}, x_{2}$ belonging to an element $M \in \Pi_{\delta}$ with $\mu(M)>0$. It follows that $f(x)$ is measurable $(\mathscr{H})$.

I am indebted to Mr. J. E. Moyal for suggesting some improvements in the presentation of the results of this paper. 


\section{References}

[1] Birkhoff, G., Integration of functions with values in a Banach space, Trans. Amer. Math. Soc. 38 (1935), $357-378$.

[2] Finch, P. D., The theory of information and statistical inference. To appear in Jour. App. Prob.

[3] Finch, P. D. A lemma on Maximal sets and the theorem of Denjoy-Vitali, this Journal 4 (1964), $195-201$.

[4] Fróchet, M., Sur l'intégrale d'une fonctionelle étendue à un ensemble abstrait, Bull. Soc. Math. France, 43 (1915), 249-267.

[5] Hildebrandt, T. H., Integration in Abstract Spaces, Bull. Amer. Math. Soc. (1953), $111-139$.

[6] Kelley, J. L., General Topology. D. van Nostrand. (1955).

Australian National University,

Canberra. 